\title{
Covariant density functional theory: predictive power and first attempts of a microscopic derivation
}

\author{
Peter Ring ${ }^{1,2, *}$ \\ ${ }^{1}$ Physics Department, Technical University of Munich, 85748 Garching, Germany \\ ${ }^{2}$ School of Physics, Peking University, Beijing 100871, People's Republic of China
}

\begin{abstract}
We discuss systematic global investigations with modern covariant density functionals. The number of their phenomenological parameters can be reduced considerable by using microscopic input from ab-initio calculations in nuclear matter. The size of the tensor force is still an open problem. Therefore we use the first full relativistic Brueckner-Hartree-Fock calculations in finite nuclear systems in order to study properties of such functionals, which cannot be obtained from nuclear matter calculations.
\end{abstract}

\section{Introduction}

In recent years important progress has been made in the ab-initio studies of nuclear properties. However, because of the tremendously growing dimensions of the underlying configuration spaces the majority of heavy nuclei are, so far, only accessible through nuclear density functional theory (DFT). Symmetries play an essential role in the construction of such functionals. One of the underlying symmetries of QCD is Lorentz invariance and therefore covariant density functional theory (CDFT) are of particular interest in nuclear physics.

As in the non-relativistic case, over the years the number of such phenomenological functionals has increased considerably. In recent years systematic investigations have been carried out to study the behaviour of the different types of functionals in regions far from stability with large neutron excess and in the area of super-heavy elements [1]. It has been found that the increase of the theoretical uncertainties in areas far from the valley of stability is connected in many cases with the single particle structure. It also well known that tensor terms have a considerable influence on such quantities [2] and that these terms are hard to determine from experimental data.

On the other hand, ab-initio calculations in nuclear matter cannot teach us much about tensor terms in density functionals, because they are usually based on spin-saturation. In order to obtain such information one has to carry out ab-initio calculations for finite nuclei. The Brueckner-Hartree-Fock (BHF) theory is one of the most promising theories for such a purpose. It provides in a fully microscopic way a density dependent effective force for mean field calculations in nuclei. Most of the investigations based on BHF-theory are devoted to infinite nuclear matter. In the seventies much effort has been used to carry out such calculations also in finite nuclei. However such non-relativistic calculations usually failed to reproduce saturation, because of the missing three-body forces. In the eighties it has been found that

\footnotetext{
*e-mail: ring@ph.tum.de
} 
relativistic Brueckner-Hartree-Fock (RBHF) theory can describe saturation in nuclear matter without tree-body forces [3].

Recently the first full RHBF calculations have been carried out for finite nuclear systems $[4,5]$. They provide a basis to determine in future the size of the tensor forces in covariant density functionals on a microscopic basis.

\section{Phenomenological and Semi-microscopic CDFT's}

In Coulombic system an essential input for the derivation of microscopic functionals is the exact numerical solution of the homogeneous electron gas at various densities. Starting from this energy functional $E[\rho]$ additional gradient terms and many other corrections have been added with great success. It seems to be reasonable to apply a similar concept in nuclear physics $[6,7]$. Of course, at present there are no exact solutions of the nuclear matter problem available. One has to rely on approximate solutions, such as sophisticated variational calculations or modern Brueckner-Hartree-Fock methods.

The basic idea of microscopic covariant density functionals is therefore to start from RBHF-calculations in nuclear matter [8] of various densities and to map the results to a Walecka model with density dependent parameters $[9,10]$. However, this mapping is not unique and therefore there are many open questions until today. In addition, none of these fully microscopic functionals is able reach the accuracy necessary for practical applications.

Much more successful are semi-microscopic functionals, which use microscopic calculations in nuclear matter in order to reduce the number of phenomenological parameters considerably. An example is the parameter set DD-ME $\delta$ [11]. Here the density dependence of the corresponding meson-nucleon couplings is adjusted to microscopic calculations in nuclear matter and only four phenomenological parameters $g_{\sigma}\left(\rho_{\text {sat }}\right), g_{\sigma}\left(\rho_{\text {sat }}\right), g_{\rho}\left(\rho_{\text {sat }}\right)\left(\rho_{\text {sat }}\right.$ is the saturation density), and $m_{\sigma}$ are fine tuned by a fit to masses and radii of finite nuclei, .

Extended applications of this functional on a global scale [1] have shown that the parameter set DD-ME $\delta$ it is comparable with all the modern relativistic high precision functionals containing considerably more phenomenological parameters. The deviations between theoretical and experimental binding energies are well below $0.5 \%$, with some exceptions for light nuclei. It should be emphasized, that there are also successful non-relativistic semimicroscopic functionals based on the same concept [7].

We can conclude that, at present, we are able to derive very successful semi-microscopic covariant density functionals [11] from ab-initio results for nuclear matter and neutron matter, containing only few remaining parameters for a fine tuning.

Table 2 shows the rms deviations for nuclear masses and radii between CDFT calculations and the experimental data for 835 even-even nuclei. Several types of covariant density functionals are used, NL3* for non-linear meson couplings, DD-ME2 and DD-ME $\delta$ for density dependent meson couplings, and DD-PC1 and DD-PK1 for density dependent point coupling models. The last line contains also rotational corrections [16] obtained in a microscopic way with the same parameters. Such beyond mean field corrections improve considerably the agreement with experiment.

\section{The problem of tensor forces}

We have to emphasize that all the microscopic input comes at this stage from ab-initio calculations in nuclear matter. On the other side, there are problems in finite nuclei, where simple density functionals of this type fail consistently. An example are single-particle energies and their distribution. This has been found in an indirect way by global investigations in the area 
Table 1. The number of phenomenological parameters $n$ together with the rms deviations for masses and radii for typical covariant density functionals [1]. See text for details.

\begin{tabular}{|l|c|c|c|}
\hline CEDF & $n$ & $\Delta E_{r m s}(\mathrm{MeV})$ & $\Delta r_{c}^{r m s}(\mathrm{fm})$ \\
\hline NL3* [12] $^{*}$ & 6 & 2.96 & 0.0283 \\
DD-ME2 [13] & 8 & 2.39 & 0.0230 \\
DD-ME $\delta$ [1] & 4 & 2.29 & 0.0329 \\
DD-PC1 [14] & 10 & 2.01 & 0.0253 \\
PC-PK1 [15] & 10 & 2.58 & - \\
PC-PK1 + E corr $[16]$ & & 1.24 & - \\
\hline
\end{tabular}

of transitional nuclei [1], which depend in some cases in a sensitive way on the single particle structure, but also in experimental observations of systematic shifts of single particle energies in isotopic chains. A famous case is the splitting of $1 h_{11 / 2}$ and $1 g_{7 / 2}$ proton configurations in the Sn-region [17], which shows a very characteristic behavior as a function of the neutron number. None of the conventional density functionals can reproduce this behaviour on the Hartree level. Only relativistic Hartree-Fock calculations which contain tensor forces in the exchange terms can reproduce this behavior. However, the strength of the tensor has to be fitted in a phenomenological way [2]. Similar results have also been found in density dependent Relativistic Hartree-Fock (RHF) theory [18].

On the other side, it is an open question, whether these shifts in observed single particle energies are an indication of the necessity of effective tensor forces in relativistic density functionals. In fact, recently it has been shown by A. Afanasjev and E. Litvinova [19] that a large part of the shifts of the $1 h_{11 / 2}$ and $1 g_{7 / 2}$ proton configurations in the Sn-region [17] can be described with the simple functional NL3* going beyong mean field and including quasiparticle-vibrational coupling [20] .

\section{Microscopic RBHF calculations in finite nuclei}

The concept to derive density functionals from properties of infinite nuclear matter cannot teach us much when it comes to properties of the functionals, which do not show up in nuclear matter calculations as for instance the tensor term in spin non-saturated systems. Here one needs ab-initio calculations in finite nuclei. An obvious way to carry out such investigations is Brueckner theory, the mother of modern density functional theory in nuclei. The advantage of relativistic Brueckner theory is that one does not need three-body forces to get saturation in nuclear matter close to the experimental area. In most of these applications the local density approximation (LDA) has been used, i.e. in a first step the self-energies are calculated in nuclear matter at various densities. In a second step the relativistic Hartree-Fock equations for Walecka-type functionals are solved in finite nuclei with density dependent coupling constants adjusted to the results of nuclear matter calculations at the corresponding density. This provides a mapping of the microscopically obtained nuclear matter results onto RHF-models of Walecka type.

Although such calculations are successful, they cannot reach the accuracy of present day phenomenological covariant density functionals. In particular, the mapping is not unique and therefore the results of different groups deviate form each other considerably. Therefore we 
started a new project to solve the RBHF equations in a large finite basis. First we started in an oscillator basis [21], but then we found that a large Dirac-Woods-Saxon (DWS) basis is more appropriate [22]. There are basis states with positive and negative energies and cut-offparameters are introduced for the basis, such that convergence is achieved. For details see Ref. [5].

Table 2. Ground state properties of ${ }^{16} \mathrm{O}$ obtained with RBHF theory are compared various other approximations and with experiment (from Ref. [4]).

\begin{tabular}{|c|c|c|c|c|}
\hline & $E(\mathrm{MeV})$ & $r_{c}(\mathrm{fm})$ & $r_{m}(\mathrm{fm})$ & $\Delta E_{\pi 1 p}^{l s}(\mathrm{MeV})$ \\
\hline EXP & -127.6 & 2.70 & $2.54(2)$ & 6.3 \\
\hline RBHF, Bonn $A$ & -113.5 & 2.56 & 2.42 & 5.4 \\
\hline RBHF (DWS) [5] & -120.7 & 2.52 & 2.38 & 6.0 \\
\hline DDRHF, PKO1 [18] & -128.3 & 2.68 & 2.54 & 6.4 \\
\hline DDRHF, PKA1 [23] & -127.0 & 2.80 & 2.67 & 6.0 \\
\hline BHF [24], AV18 & -134.2 & & 1.95 & 13.0 \\
\hline $\mathrm{CC}[25], \mathrm{N}^{3} \mathrm{LO}$ & -120.9 & & 2.30 & \\
\hline NCSM [26], N³ LO & $-119.7(6)$ & & & \\
\hline NLEFT [27], N²LO & $-121.4(5)$ & & & \\
\hline
\end{tabular}

As an application we consider the nucleus ${ }^{16} \mathrm{O}$. We use the realistic NN interaction Bonn A which has been adjusted to the NN scattering data in Ref. [28]. The ground-state properties are listed in Table 2. The results of our full RBHF calculation are compared with the corresponding experimental data and with several other calculations: BHF is a non-relativistic Brueckner calculation based on the interaction Bonn A. We also show results obtained in RHF-calculations with the phenomenological effective interaction PKO1, which has been fitted to binding energies and charge radii of a set of spherical nuclei. It is seen that the ground-state properties in RBHF theory are improved considerably as compared with the non-relativistic results. This energy of ${ }^{16} \mathrm{O}$ is also very close to the value of $E=-119.7 \mathrm{MeV}$ obtained in the No Core Shell Model (NCSM) calculation using the chiral $N N$ interaction $\mathrm{N}^{3} \mathrm{LO}$ [26]. Of course the results of the calculations with PKO1 which has been fitted to these data shows only a very small deviation from the experimental values.

\section{Conclusions}

In summary, the full relativistic Brueckner-Hartree-Fock (RBHF) equations have been solved in a Dirac-Woods-Saxon (DWS) basis. The relativistic structure of the two-body matrix elements as well as of the Pauli operator is fully taken into account. The only input is the bare $N N$-interaction Bonn A adjusted to the scattering phase shifts in Ref. [28]. No other parameter is used. Since nuclear matter calculations within the same framework produce results far away from the Coester line and close to the experimental values of saturation, we neglect at this stage three-body forces.

Despite the good agreement of these results, there is room for improvements. The RBHFtheory presented here is no exact solution of the nuclear many-body problem. So far, rearrangement terms are not taken into account and higher order diagrams in the hole-line expansion are not included. 
On the other side, our method has the potential to investigate heavier nuclei, where exact solutions are impossible, in particular systems without spin saturation and with large neutron excess. In this case we hope to be able to gain a parameter-free, microscopic understanding of open questions in modern phenomenological density functional theories, such as their isospin dependence or the importance of the tensor terms. As an example it has been found that the spin-orbit splitting in neutron droplets shows a very characteristic behavior as a function of the neutron number, which can be reproduced in phenomenological functionals with tensor terms of an appropriate size [29].

\section{Acknowledgements}

This work was partly supported by the DFG cluster of excellence "Origin and Structure of the Universe" (www.universe-cluster.de).

\section{References}

[1] S.E. Agbemava, A.V. Afanasjev, D. Ray, P. Ring, Phys. Rev. C 89, 054320 (2014)

[2] G.A. Lalazissis et al, Phys. Rev. C 80, 041301 (2009)

[3] M.R. Anastasio, L.S. Celenza, C.M. Shakin, Phys. Rev. Lett. 45, 2096 (1980)

[4] S.H. Shen, H.Z. Liang, J. Meng, P. Ring, S.Q. Zhang, Phys. Rev. C 96, 014316 (2017)

[5] S.H. Shen et al, Chin. Phys. Lett. 33, 102103 (2016)

[6] S. Fayans, JETP Lett. 68, 169 (1998)

[7] M. Baldo, P. Schuck, X. Viñas, Phys. Lett. B 663, 390 (2008)

[8] R. Brockmann, R. Machleidt, Phys. Rev. C 42, 1965 (1990)

[9] R. Brockmann, H. Toki, Phys. Rev. Lett. 68, 3408 (1992)

[10] F. Hofmann, C.M. Keil, H. Lenske, Phys. Rev. C 64, 034314 (2001)

[11] X. Roca-Maza et al, Phys. Rev. C 84, 054309 (2011)

[12] G.A. Lalazissis et al,Phys. Lett. B 671, 36 (2009)

[13] G.A. Lalazissis, T. Nikšić, D. Vretenar, P. Ring, Phys. Rev. C 71, 024312 (2005)

[14] T. Niksic, D. Vretenar, P. Ring, Phys. Rev. C 78, 034318 (2008)

[15] P.W. Zhao, Z.P. Li, J.M. Yao, J. Meng, Phys. Rev. C 82, 054319 (2010)

[16] Q.S. Zhang, Z.M. Niu, Z.P. Li, J.M. Yao, J. Meng, Front. Phys. p. 529 (2014)

[17] J.P. Schiffer et al, Phys. Rev. Lett. 92, 162501 (2004)

[18] W.H. Long, N. Van Giai, J. Meng, Phys. Lett. B 640, 150 (2006)

[19] A.V. Afanasjev, E. Litvinova, Phys. Rev. C 92, 044317 (2015)

[20] E. Litvinova, P. Ring, Phys. Rev. C 73, 044328 (2006)

[21] P. Ring, J. Phys. Conf. Ser. 580, 012005 (2015)

[22] S.G. Zhou, J. Meng, P. Ring, Phys. Rev. C 68, 034323 (2003)

[23] W.H. Long, H. Sagawa, N.V. Giai, J. Meng, Phys. Rev. C 76, 034314 (2007)

[24] B.S. Hu, F.R. Xu, Y.Z. Ma, Q. Wu, Z.H. Sun, Phys. Rev. C 95, 034321 (2017)

[25] G. Hagen et al, Phys. Rev. C 80, 021306 (2009)

[26] R. Roth et al, Phys. Rev. Lett. 107, 072501 (2011)

[27] T.A. Laehde et al, Phys. Lett. B 732, 110 (2014)

[28] R. Machleidt, Adv. Nucl. Phys. 19, 189 (1989)

[29] S.H. Shen, H.Z. Liang, J. Meng, P. Ring, S.Q. Zhang, Phys. Lett. B 778, 344 (2018) 